\title{
Identifying Factors Which Contribute to the Magnitude of Excess Correlations between Magnetic Field-Paired Volumes of Water
}

\author{
Nicolas Rouleau, Trevor N. Carniello, Michael A. Persinger \\ Biomolecular Sciences Program, Laurentian University, Sudbury, Canada \\ Email: mpersinger@laurentian.ca
}

Received 23 May 2016; accepted 15 July 2016; published 18 July 2016

Copyright (C) 2016 by authors and Scientific Research Publishing Inc.

This work is licensed under the Creative Commons Attribution International License (CC BY).

http://creativecommons.org/licenses/by/4.0/

c) (i) Open Access

\begin{abstract}
Excess correlations, one of the quantitative demonstrations of "entanglement", have been experimentally demonstrated as spontaneous shifts in photon properties and molecular interactions. The magnitudes of the excess correlations have been enhanced experimentally for photon emissions and proton densities in aqueous solutions when the loci containing these physical chemical reactions shared circular magnetic fields whose angular velocities were always changing. In the present experiment, quantities of spring water each placed in one of two loci (local or non-local) separated by 100 or $10,000 \mathrm{~m}$ were exposed simultaneously to toroidal magnetic fields within a paradigm that has been shown to produce conspicuous excess correlations in shifts of photon emissions, $\mathrm{pH}$ in spring water, and human brain activity as inferred by electroencephalography. The non-local area that was not injected with proton donors displayed a reliable shift in $\mathrm{pH}$ when the local area was serially injected with small aliquots of protons but only during the presentations of the field parameters known to produce "entanglement". The effect was most obvious when the global geomagnetic activity was less than $\mathrm{Kp}<3$. The probability is high that convergent similarities in the magnitudes of the local geomagnetic intensities of the two loci enhance the strength of the excess correlations. These results suggest that a minimal energy and inexpensive system, not involving classical electromagnetic transmission through a medium, but influenced by the global geomagnetic field activity, could be employed to generalize and superpose information between two non-local spaces.
\end{abstract}

\section{Keywords}

Excess Correlation, Water, Toroids, Geomagnetic Activity, Signal Detection 


\section{Introduction}

Phenomena produced by non-local mechanisms or processes are described by the excess correlations of some measurement between two loci separated by non-traditional distances [1]. Whereas phenomena restrained to local effects involve identifiable medium with specific properties through which they are propagated and are prone to some variant of the inverse square or inverse cubic attenuation over distance, non-local phenomena are not presumably restricted by spatial distances or temporal discrepancies [2]. The establishment of the manifestation of non-local phenomena at macroscopic levels would be important to chemistry, physics, and astronomy because information could be transposed and superposed over any two space-time coordinates. This could potentially alter interpretations regarding the dispersion of similarities of properties for chemical and biological systems across vast distances and durations. In the experiments reported here we showed that non-local alterations in proton densities as inferred by measureable shifts in $\mathrm{pH}$ when two loci shared the same changing angular velocity toroidal magnetic fields could be demonstrated over the furthest distance tested which was about $10 \mathrm{~km}$.

Most demonstrations of non-local phenomena have involved photons. Arnesen et al. [1] have stated "it is well known that distinct quantal systems can be correlated in a stronger then classical manner”. This phenomenon has been labelled as excess correlation or entanglement. They found that excess correlation between two spins in an antiferromagnetic solid could be increased by increasing the external field or temperature. Megidish et al. [3] showed the potential for entanglement between photons that had never co-existed when a source photon differentiated into two lower energy photons while preserving momentum and energy. Recent experiments by Fickler et al. [4] indicated that helical photons that mediate a quantized amount of orbital angular momentum may show marked entanglement between two photons differing by very large quantum numbers. The resulting quantum teleportation, as described by Olmschenk et al. [5], produces reliable transfer of quantum states.

A relaxation process is traditionally expected to produce a loss of quantum correlations. However if the systems share a common thermostat field there is entanglement in an ensemble of non-interacting atoms [6]. Julsgaard et al. [7] experimentally demonstrated entanglement in two macroscopic gas systems, each containing about $10^{12}$ atoms for about $0.5 \mathrm{~s}$. In this situation, the entanglement was produced by interaction of the atoms with polarized light. Later Dotta and Persinger [8] found that two chemoluminescent reactions that generated photons when separated by $10 \mathrm{~m}$ or $3 \mathrm{~km}$ while sharing the same circularly rotating magnetic fields with changing angular velocities displayed conspicuous excess correlation and quantitative summation whose duration was $\sim 8$ min. The estimated ratios of numbers of molecules to the duration of the entanglement were similar to that found for the caesium gas molecules reported by Julsgaard et al. [7]. The two loci behaved as if they had been superimposed upon the same space and hence the quantity of photons emitted doubled.

Dotta et al. [9] measured excess correlations between shifts in $\mathrm{pH}$ in two quantities of spring water separated by $10 \mathrm{~m}$ if both shared the same changing angular velocity circular magnetic fields as those that produced the “doubling of photons" in the chemiluminescent experiments. When small aliquots of a proton donor (a weak acid) were injected once every minute into one of the quantities of water there were very small but systematic increases in alkalinity in the other quantity of water that received no injections but shared the same temporal pattern of magnetic field application. Later calculations and experiments [10] indicated that the amount of shift in the $\mathrm{pH}$ towards alkalinity was related to the predicted quantity of loss of protons from hydronium ions (or gain in hydroxyl ions) that was proportional to the magnetic energy within the volume of water provided by the external magnetic fields.

Rouleau et al. [11] replicated these experiments that demonstrated the excess correlation between pH shifts between two quantities of water separated by about $1 \mathrm{~m}$ that shared similar temporally-patterned circularly generated magnetic fields. However the fields were generated within toroids not a ring of eight solenoids. The strengths of the time-varying fields that were most effective were within the $30 \mathrm{nT}$ range. Moreover during the presentation of the field the east-west static magnetic field component was diminished by about 1 to 5 nT. This is important intensity given such shifts have contributed to powerful biological effects as shown by St-Pierre et al. [12] and appear to be intercalated with the production of photon emissions from the cerebrums of volunteers when they are imagining white light while sitting in a hyperdark room [13] [14]. Vladimirskii and Temur'yants [15] have shown that $1 \mathrm{nT}$ fields exert significant effects upon physicochemical systems. Recently Persinger and St-Pierre [16] calculated that the energy coupled to the 5 nT geomagnetic fluctuation and variation in the Newtonian Gravitational constant within the human cerebrum was comparable to that associated with a percept and during human cognition. 
On the surface of the earth, most systems are immersed in the geomagnetic field. Korotaev et al. [17] demonstrated that quantum non-locality as reflected in non-local dependence of dissipative processes was manifested in geomagnetic variations. It may even involve advanced correlations of unknown states. These empirical measurements were consistent with the long-established non-potential component of the earth's magnetic field that is associated with an electric current system in the order of 0.1 to $10 \mathrm{nT}$ that corresponds to radial current densities between 10 and $1000 \mathrm{pA} \cdot \mathrm{m}^{-2}$ [18]. Given the clever conception by Hunter et al. [19] that the earth itself could be considered a source of polarized electrons that could mediate long-range spin-spin interactions (one of the potential mediators of entanglement) the probability that quantities of geomagnetic intensities in the two loci that share the effective experimental fields that produce excess correlation and the level of global geomagnetic activity could affect the magnitudes of these excess correlations was considered feasible. Here we present evidence to support this series of inferences, which will assist in designing further excess correlation experiments with increased technical precision.

\section{Materials \& Methods}

\subsection{Excess Correlation Equipment}

Two toroid-shaped coils with circumferences of $79.8 \mathrm{~cm}$, wrapped with 225 turns of 16 gauge copper stereo speaker wire, were constructed. Coils were sealed with black, vinyl electrical tape and equipped with jumper cable pins in order to interface with a solderless breadboard. Arduino UNO R3 microcontrollers carried patterned current from Lenovo ThinkPad laptops running Windows 7 and Arduino 1.0.6 as 3 ms point-waves to the solderless breadboards fitted with electronic components which were then directed to the toroids. Effectively, experiments always occurred in two spaces simultaneously. Both spaces were equipped with identical equipment wherein the toroid-microcontroller systems were synchronized such that EMF patterns directed to the respective spaces trough the devices were simultaneous.

Two patterns were employed throughout trials. The first, the Primer field, consisted of 3 ms pulses separated in time by $20 \mathrm{~ms}$ pauses which increased with each successive pulse by $2 \mathrm{~ms}$ where the frequency of the pulses decreased until the 7th pulse, after which the pattern was re-initiated and looped continuously for 360 seconds. The second field pattern, the Effector field, consisted of 3 ms pulses separated in time by 20 ms pauses which decreased with each successive pulse. In contrast to the Primer pattern, the pauses between each pulse within the Effector pattern decreased in duration with each successive pulse by 2 ms such that the frequency increased until the 7th pulse. The Effector pattern looped continuously for 720 seconds. Each trial consisted of 1080 seconds of field exposure with the Primer sequence always preceding the Effector sequence. For the purposes of analysis and investigation, the Effector sequence can be thought of as consisting of an early-phase (Effector 1) and a late-phase (Effector 2) component. These sequences which will at times be treated separately are in fact two time periods within the same field exposure sequence and not distinct or independent exposures.

Detailed descriptions of this equipment including investigations into the local background field fluctuations as a consequence of the applied field patterns have described in detail by Rouleau\&Persinger [20]. Detailed video instructions on how to build and operate the equipment can be accessed at excesscorrelation.net.

\section{2. pH Measurement Equipment}

The $\mathrm{pH}$ measurements were performed using glass $\mathrm{pH}$ probes coupled to DrDAQ boards where data streamed to a Lenovo ThinkPad laptop computer within the Pico Technology (UK) program interface. The sampling rate of the device was set to $1 \mathrm{~Hz}$ and pre-programmed to terminate data collection after 1080 seconds had elapsed. Probes were regularly calibrated with standardized solution and pre-trial baseline pH levels were verified within the 7.00 to 7.50 range.

\subsection{Magnetometer Recordings}

The three-dimensional sensor of a MEDA FVM-400 Vector Magnetometer was positioned within the center of the toroid space in order to measure background fluctuations in the intensity (nT) of the local EM environment. The X-axis of the probe was oriented within the North-South plane with reference to $0^{\circ}$ declination or Magnetic North. This orientation positioned the Y-axis within the East-West orientation and the Z-axis within the vertical plane. Recordings $(n=9)$ were performed at 1300 local time, each consisting of 5 minutes of data recorded at a 
sampling frequency of $1 \mathrm{~Hz}$. Data were streamed to a Lenovo ThinkPad laptop computer running Windows 7 positioned 2 meters away within the S-W direction relative to the sensor's oriented axes.

\subsection{Procedure}

The procedure employed here was similar to that which was reported by Rouleau et al. [11]. The experiments were conducted within three testing spaces: Location A, B, and C. Every trial was performed in tandem by two experimenters which were situated within one of the three spaces. Two configurations were assigned. The first involved one experimenter within Location A and another within Location B, separated by $10^{4} \mathrm{~m}(10 \mathrm{~km})$. The second configuration involved experimenters positioned within Locations B and C, separated by $10^{2} \mathrm{~m}$. No trials were performed which simultaneously involved Location A and C. In both the A-B and B-C configurations, both spaces contained the same experimental equipment.

Unless specified otherwise, all procedures are identical within both testing spaces. A $250 \mathrm{~mL}$ beaker containing $25 \mathrm{~mL}$ of spring water ( $4 \mathrm{mM}$ of $\mathrm{HCO}_{3} ; 1.77 \mathrm{mM} \mathrm{Ca} ; 76 \mu \mathrm{M}$ of $\mathrm{Cl} ; 1.3 \mathrm{mM}$ of Mg; $41.9 \mu \mathrm{M}$ of $\mathrm{NO}_{3} ; 61 \mu \mathrm{M}$ $\mathrm{SO}_{4} ; 17.9 \mu \mathrm{M} \mathrm{K} ; 43.5 \mu \mathrm{M} \mathrm{Na}$ ) was placed within the center of the toroid. To begin the trial, the Primer field was initiated by uploading the pattern to the microcontroller and the $\mathrm{pH}$ probe was set to record. The $\mathrm{pH}$ probe recorded throughout the entire 1080 second period without interruption. The Effector field was initiated after 360 seconds of Primer field exposure. The Effector field looped continuously until 1080 seconds had elapsed and pH data was no longer being sampled.

Though most procedures are identical within both testing spaces, there were a few exceptions. The "local" and "non-local" spaces were designated at the outset of the experiment. The local space would be associated with injections $(50 \mu \mathrm{L})$ of a weak proton donor ( $5 \%$ acetic acid) into the beaker at fixed increments throughout the trial (i.e. minute 4 as well as minutes 7 - 15). The non-local space was not manipulated in this way. The beaker and its contents were not disturbed throughout the course of a given trial by the experimenter. Location $\mathrm{B}$ served as the "local" space in both the A-B and B-C configurations. This meant that injections were only performed within Location B.

\section{Results}

\subsection{Experimental Demonstration of Excess Correlation}

Paired t-tests revealed increased average absolute Pearson $\mathrm{r}$ between $\mathrm{pH}$ measurements during the Effector 1 sequence relative to the Primer sequence when both spaces received a field exposure, $\mathrm{t}(8)=2.33, p<0.05, \mathrm{r}^{2}=$ 0.40 (Figure 1, Both On). Whereas when both spaces were exposed to the EMF application they demonstrated excess correlation exceeding that which would be expected due to chance alone, when only one location was

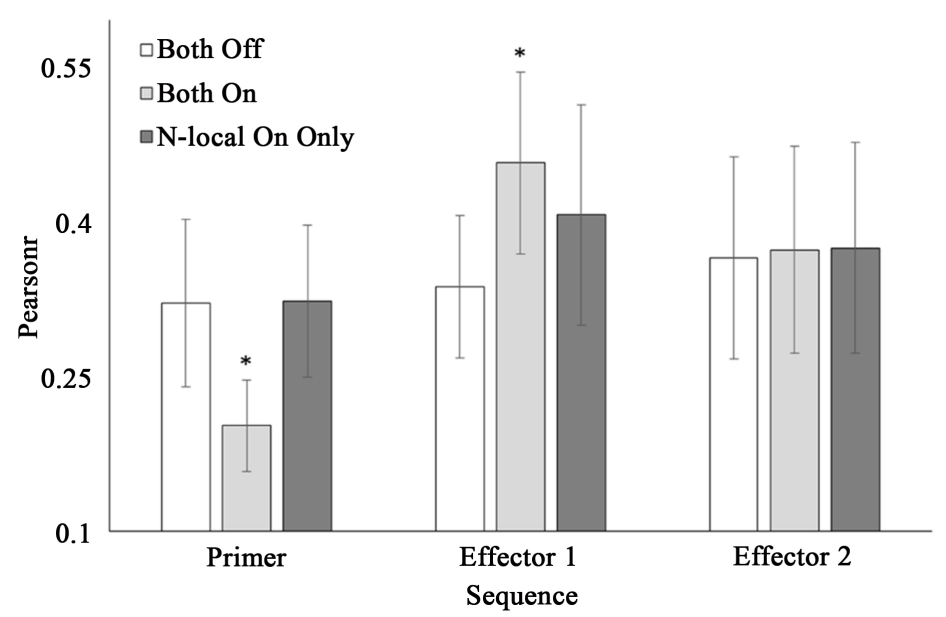

Figure 1. Means and SEMs of absolute Pearson $r$ as a function of the excess correlation protocol demonstrating increased strength of the association between $\mathrm{pH}$ values $(\mathrm{n}=360)$ during the Effector 1 sequence relative to the Primer sequence when both beakers are exposed to identical EMF patterns. Significant differences within the Both On condition are indicated. 
exposed to an EMF the magnitude of the correlation remained fixed over time within trials, $t(8)=0.86, p=0.42$ (Figure 1, N-local On Only). Similarly, if both spaces were unexposed to EMFs, no differences could be discerned between sequences, $\mathrm{t}(8)=0.22, p=0.83$ (Figure 1, Both Off).

Instead of dividing trial periods into three equal $360 \mathrm{~s}$ increments (i.e. Primer, Effector 1, and Effector 2), data were segmented into 11 equal 100 second increments (e.g. 0 - 100 s, $100-200$ s, etc.) and absolute Pearson r values were computed. When comparing the absolute Pearson $r$ values associated with the first increment of time (i.e. 0 - $100 \mathrm{~s}$ ) to those for every subsequent increment of time (i.e. $100-200 \mathrm{~s}$ to $1000-1100 \mathrm{~s}$ ) within EMF exposure conditions, it was apparent that when both sites were exposed to the excess correlation procedure (Both On), significant differences could be identified. These relative differences are visualized in Figure 2 which plots t-statistic values (y-axis) as a function of each comparison (x-axis). If the sites were unexposed (Both Off) or partially exposed (N-local On Only), no significant differences were identified over the course of the procedure $(p>0.05)$.

The "switch" increment (i.e. 300 s - 400 s), where the Effector Field known to produce the excess correlation in quantitative changes in reactions between two non-traditional localities [8]-[11], was marked by an increased average absolute Pearson $\mathrm{r}$ value relative to the first increment (i.e. 0 - 100), $\mathrm{t}(8)=2.65, p<0.05, \mathrm{r}^{2}=0.47$. A marginal increase was also noted when comparing the first increment to the 10th increment (i.e. 900 - 1000), $\mathrm{t}(8)$ $=2.28, p=0.052, r^{2}=0.39$. These comparisons are highlighted in Figure 3.

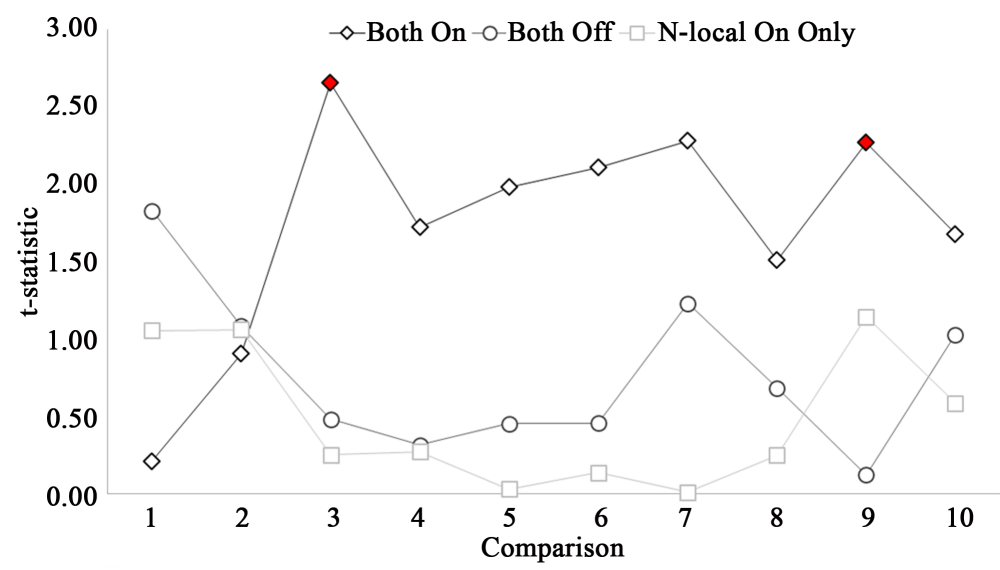

Figure 2. t-statistics associated with sequential comparisons of within-condition absolute Pearson $\mathrm{r}$ values relative to the $0-100$ second period presented in Figure 1 . Significant differences with $p<0.05$ are indicated by filled markers.

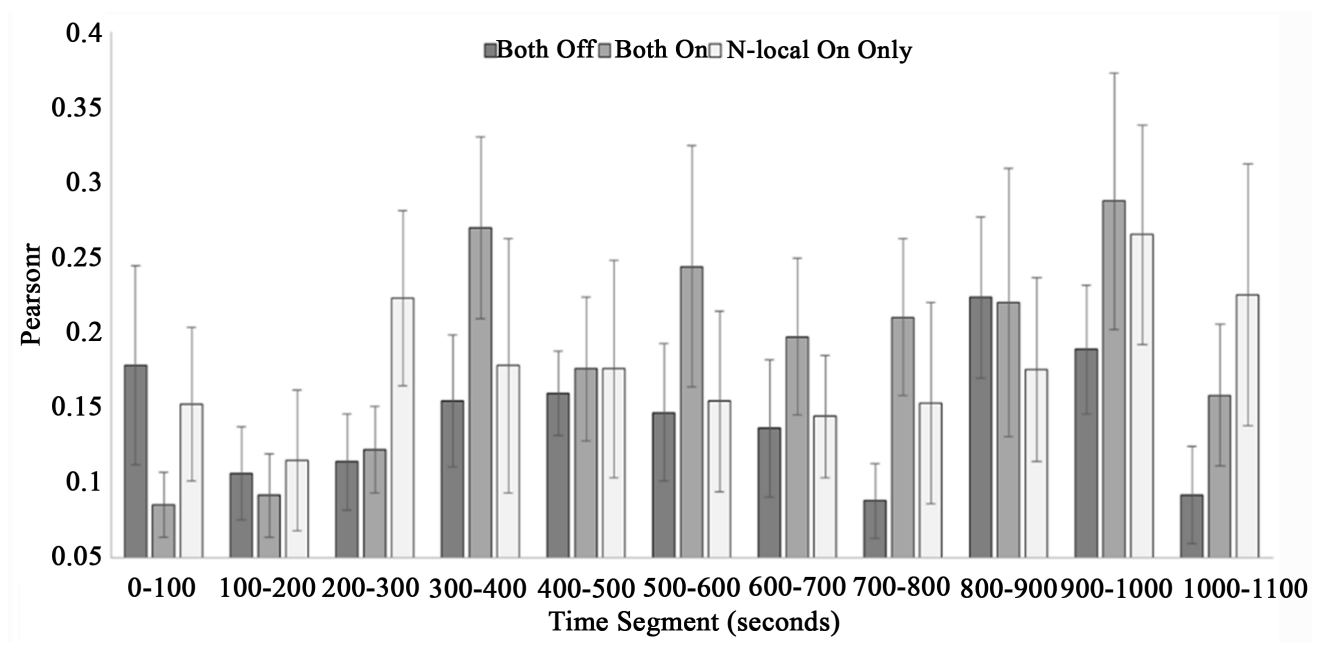

Figure 3. Pearson $\mathrm{r}$ (y-axis) as a function of time segment (x-axis) for each experimental condition. The time segment within which the "switch" from Primer to Effector sequence is indicated by the asterisk. 


\subsection{Contributions of Extrinsic Electromagnetic Field Sources}

Multiple ANOVAs revealed differences in the magnitude of the static geomagnetic field along X- (North-South, $p<0.001)$ and Z-axes (vertical, $p<0.005)$ in addition to the resolved field $(p<0.005)$ as a function of the location. The results are shown in Figure 4. The values for the Y-axis (East-West) geomagnetic intensity did not differ as a function of location $(p>0.05)$. These values are shown in Figure 5. For the purposes of this project, only two of the possible three combinations of locations were relevant to the analysis: Location A \& B (separated by $10^{4} \mathrm{~m}$ ), and Location B \& C (separated by $10^{2} \mathrm{~m}$ ). Differences were not noted between Locations A \& $\mathrm{C}(p>0.05)$ though no trials were completed between these spaces.

When testing days associated with low geomagnetic activity as indicated by low maximum Planetary K-index values $(\mathrm{K}<3)$ over the course of the 24 hour period were selected, ANOVAs revealed between-condition differences during the $300-400$ ( 5 to $6.6 \mathrm{~min})$ second increment $\left(\eta^{2}=0.69, \mathrm{p}<0.05\right)$ in addition to the $700-800$ second increment $\left(\eta^{2}=0.87, p<0.005\right)$. Only differences within the latter increment remained statistically significant after a Bonferroni correction $(\alpha=0.005)$. These between-condition differences were not obtained when all cases entered into the analysis including those associated with $K$ values of $3(n=6)$ and $4(n=4)$. The primary sources of variance were increases in absolute Pearson $r$ during the Both On condition relative to the Both Off condition $(p<0.05)$. This suggested that during the Effector 2 period, $~ 7.5$ minutes after the initiation of the Effector field, fluctuations in $\mathrm{pH}$ within the spatially isolated (non-local) volumes of water became coherent to a greater degree when simultaneously exposed relative to when unexposed. In other words the excess correlation was increased when the global geomagnetic activity was less.

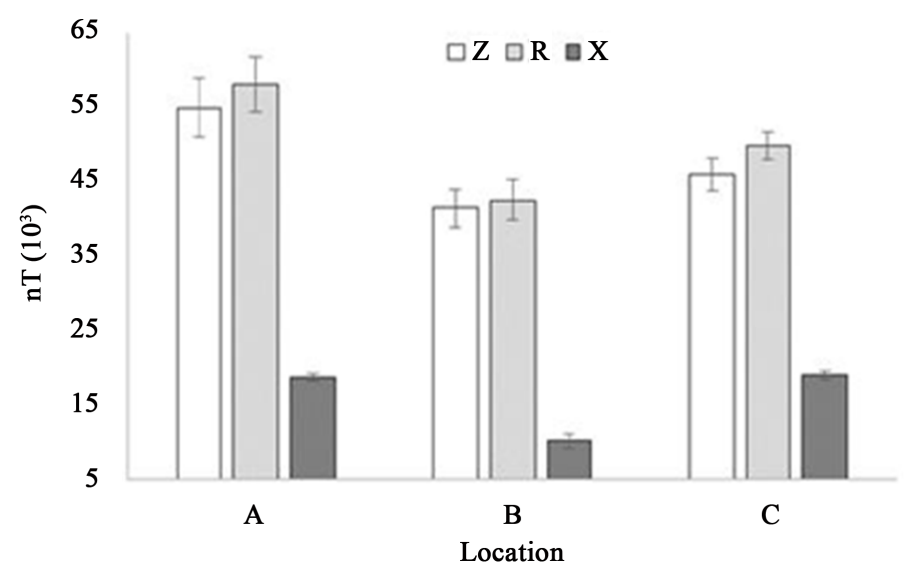

Figure 4. Vertical (Z), N-S (X) and resolved (R) field differences as a function of the testing space (Location).

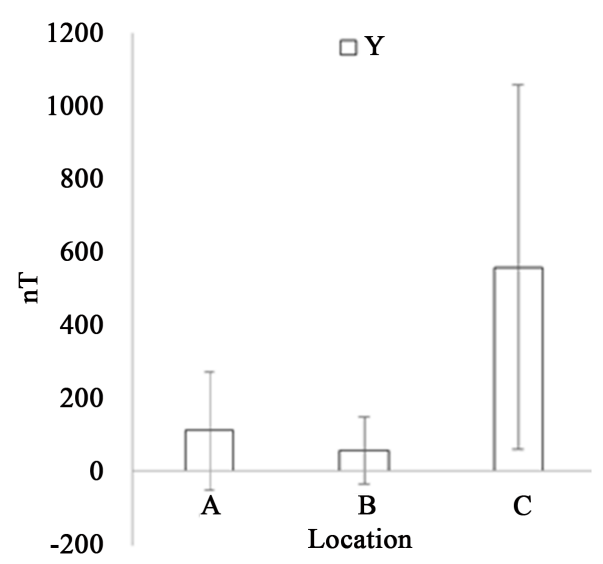

Figure 5. Means and SEMs (vertical bars) for the intensity (in nanoTesla) for the East-West (Y) component within the experimental areas within which the excess correlation experimental fields were generated. 
Figure 6 compares two trials during the Both On condition, one recorded during very low geomagnetic storm conditions where $K=1$, and one recorded during moderate geomagnetic storm conditions where $K=4$. Significant increases in the magnitude of the excess correlations $(\mathrm{n}=360, \mathrm{z}>1.96, p<0.05)$ during the K-1 trial relative to the K-4 trial for the 200 - 300, 300 - 400, 700 - 800, 800 - 900, and 900 - 1000 time increments were apparent. In this particular instance, a single significant decrease was noted within the first 100 second increment $(\mathrm{z}=2.0, p<0.05)$ though this did not remain consistent when incorporating further trials $(p>0.05)$.

Independent t-tests revealed increased Pearson $r$ values associated with the Both On condition $(\mathrm{M}=0.42$, $\mathrm{SEM}=0.13)$ relative to the Both Off condition $(\mathrm{M}=0.11$, SEM $=0.04)$ during the $300-400$ second increment when $\mathrm{K}<3, \mathrm{t}(5)=3.36, p<0.05, \mathrm{r}^{2}=0.69$. The effect persisted into the $400-500$ second increment when $\mathrm{K}<$ 3 with an absolute Pearson $r$ difference of $.23\left(\mathrm{r}^{2}=0.61, p<0.05\right)$. After dissipating during the $500-600$ second increment $(\mathrm{p}=0.09)$ and the $600-700$ second increment $(p=0.23)$, the effect re-emerged during the $700-800$ second increment, $\mathrm{t}(5)=5.67, p<0.005, \mathrm{r}^{2}=0.87$. This latter difference, despite a very limited sample size due to case selection $(\mathrm{n}=3)$, was also identified when comparing the Both On condition $(\mathrm{M}=0.37, \mathrm{SEM}=0.06)$ and the N-Local On Only condition $(\mathrm{M}=0.04$, SEM $=0.04)$ with $\mathrm{K}<3, \mathrm{t}(2)=-4.482, \mathrm{p}<0.05, \mathrm{r}^{2}=0.91$. No significant differences in absolute Pearson $\mathrm{r}$ were detected when comparing Both Off and N-Local On Only conditions $(p>0.05)$.

Differences in absolute Pearson $r$ as a function of the interaction between the spaces where the trials were held, the experimental condition, and the geomagnetic storm level of the time were investigated next. These results would elucidate the potential relationship between the interacting EMFs of the toroids, the geomagnetic sphere, and those of local electronics and power supplies which contributed to the local EM environment. It should be noted that, although recorded field intensities differed between locations, there were implicit distance differences between the two paired locations where Location A \& B were separated by $10^{4} \mathrm{~m}$ and Location B \& C are separated by $10^{2} \mathrm{~m}$.

Simple effects of location (i.e. paired spaces of testing) were identified where average absolute Pearson $\mathrm{r}$ values associated with trials involving Location A \& B $(M=0.12$, SEM $=0.04)$ were decreased relative to those associated with Location B \& C $(M=0.39$, SEM = 0.04) during the $700-800$ second segment (Effector 2 sequence), $\mathrm{t}(7)=-4.73, p<0.005, \mathrm{r}^{2}=0.76$. The effect size increased when selecting for trials associated with 24 hour maximum Kp values below 4, $\mathrm{r}^{2}=0.83$, though the difference in absolute Pearson remained consistent $\left(\mathrm{r}_{\text {Diff }}=0.26\right)$. Differences as a function of location could not be discerned for Both Off and N-Local On Only conditions during any of the discrete time segments $(p>0.05)$.

\subsection{Intrinsic Variability within the Observed System}

Dynamic shifts in the central tendency, such as the mean, in an aggregate usually begin with an influence upon the most responsive units of the aggregate. This produces an increase in the dispersion of values, or variability, around the mean. As a result, initial effects or "weak" effects that are not sufficient to separate the means of the

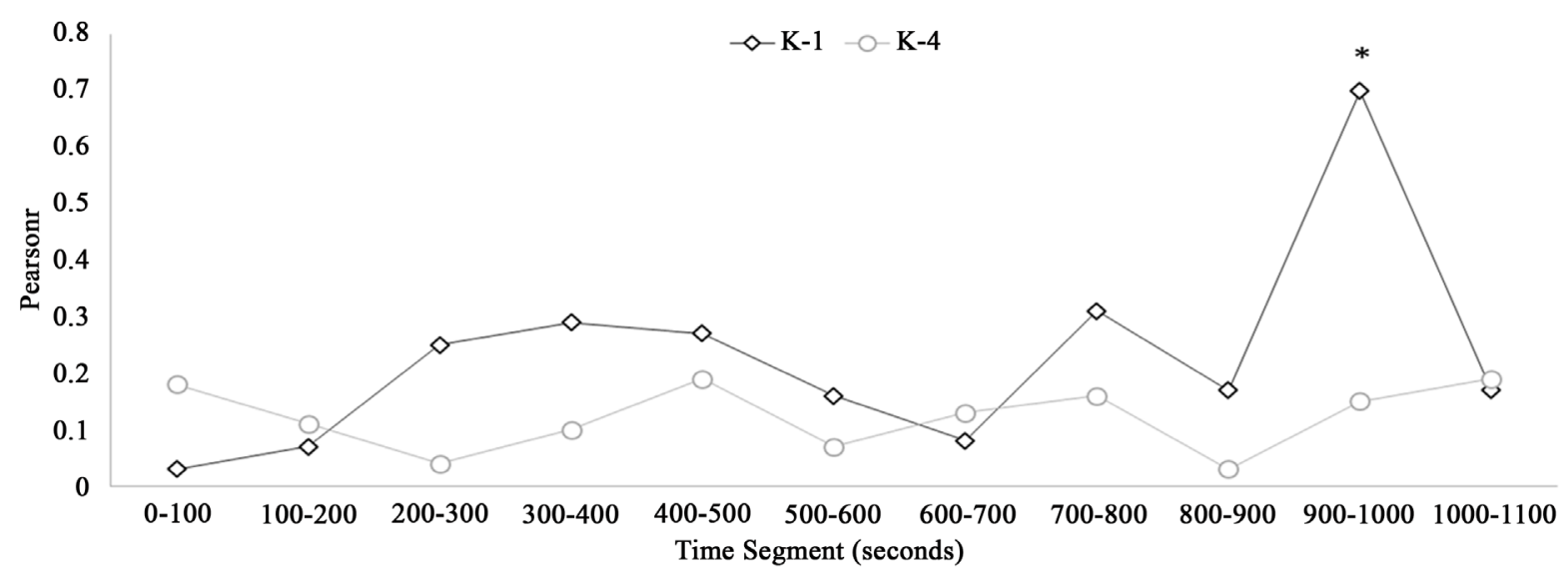

Figure 6. Correlation (absolute Pearson r) between $\mathrm{pH}$ values obtained within the two volumes of water for individual trials with Kp 24-Max of 1 (diamonds, K-1) and 4 (circles, K-4) when both beakers are exposed to the EMF sequences of the excess correlation protocol (Both On) as a function of time. 
experimental and reference group in a statistically significant manner, may be manifested only by increases or decreases in the statistic for dispersion such as standard deviation or variance. From some perspectives the absence of a significant difference between the means of two populations but the emergence of differences in variability sets the conditions for the maintenance of "subthresold" or stochastic processes through which information can be mediated. When there are no statistically significant differences between the means but there is a significant difference between the variances of the two means, then stochastic processes could operate within a relatively narrow band of quantity compared to the measurements that reflect the mean.

Logarithmic relationships were identified between the coefficient variation (CV) of the $\mathrm{pH}$ of spring water within the non-local beaker (i.e. the space where no material is injected into the beaker) over the course of a given trial and the correlation between the $\mathrm{pH}$ of the local (i.e. injection site) and non-local sites during the Effector $1(r=0.88, p<0.001)$ and Effector $2(r=0.67, p<0.05)$ sequences. The Primer sequence did not demonstrate a logarithmic or linear relationship of this kind. Furthermore, the $\mathrm{CV}$ of the $\mathrm{pH}$ of the local beaker (i.e. injection site) did not systematically relate to the correlation between the $\mathrm{pH}$ of both spaces $(p>0.05)$. These relationships are aggregated in Figure 7.

The observed relationships were without regard to condition, however, when examining the Effector 2 sequence it was apparent that local EMFs were driving the effects. Figure 8 shows logarithmic relationships between the $\mathrm{CV}$ of the non-local beaker's $\mathrm{pH}$ values and the Pearson $\mathrm{r}$ indicative of local-non-local beaker $\mathrm{pH}$ during the Effector 2 sequence for Both On $(r=0.94)$ and N-Local On Only $(r=0.94)$ conditions but not Both Off $(r=0.04)$-the only condition wherein no EMF was applied to the non-local beaker.

\section{Discussion}

The empirical test for the validity that entanglement has occurred is the experimental demonstration of excess correlations between two loci containing dynamics processes that are evident independent of distance. The general approach to entanglement is that when two systems share a state a proportion of that state remains in spacetime. This is very similar to the concept of memory when two sets such as A and B become proximal in spacetime, A still maintains a subset of B and B still maintains a subset of A long after this contiguity has attenuated. We suggest that the actual physical mechanisms by which entanglements occur, like other phenomena, should
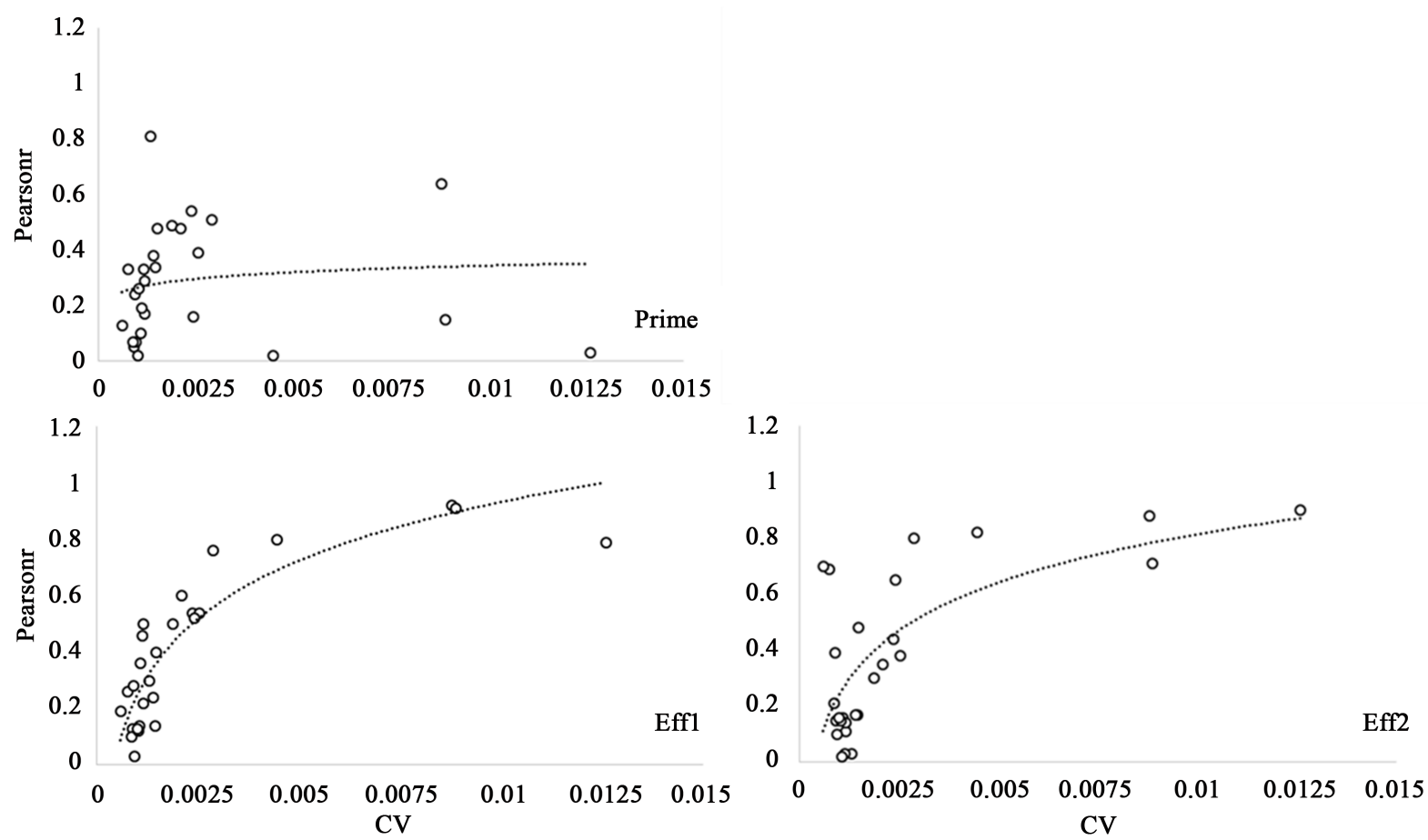

Figure 7. Pearson $\mathrm{r}$ for $\mathrm{pH}$ of paired beakers and $\mathrm{CV}$ of non-local beaker $\mathrm{pH}$ during Primer (top left), Effector 1 (bottom left), and Effector 2 (bottom right) sequences when including all experimental conditions. 

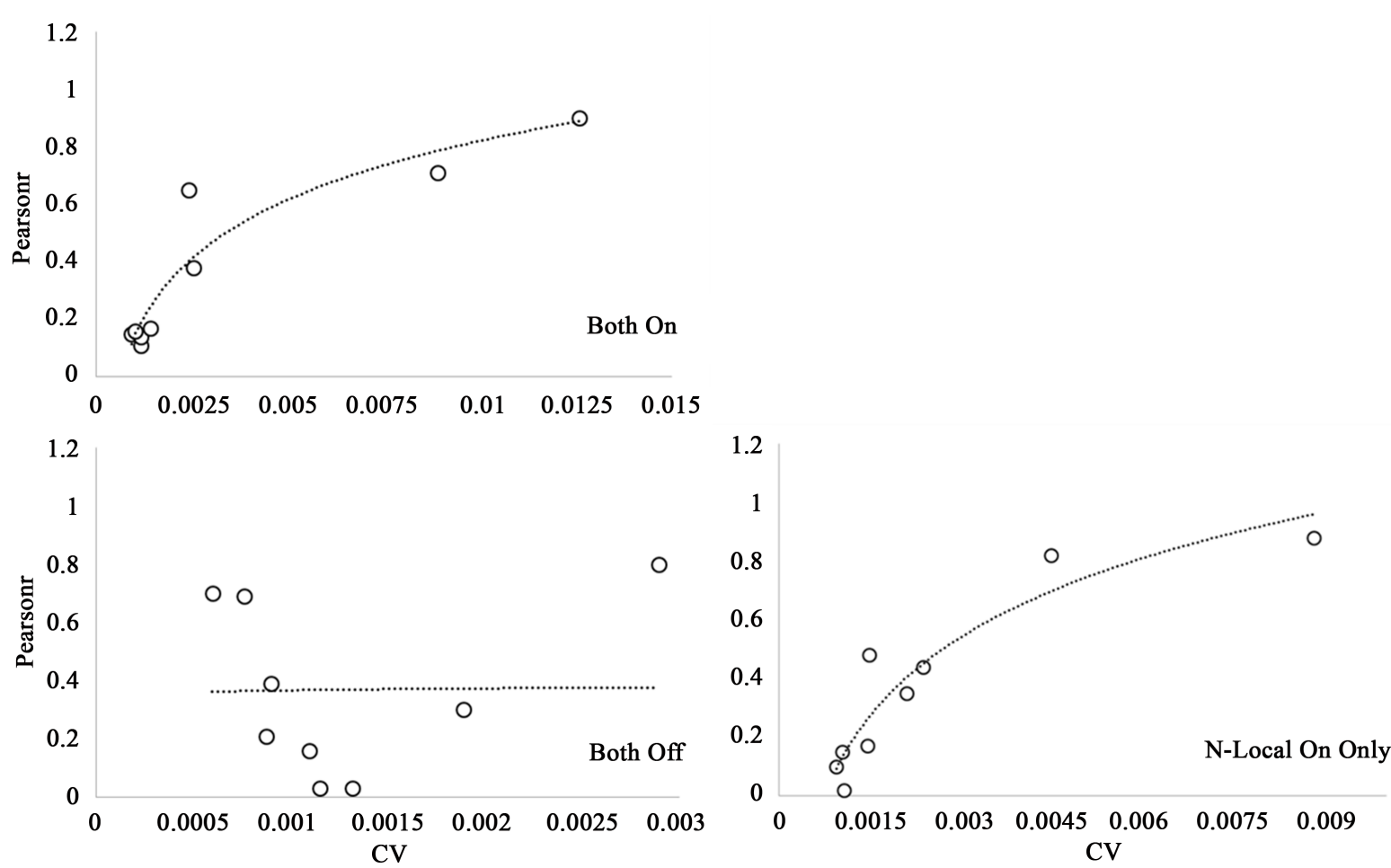

Figure 8. Pearson $\mathrm{r}$ for $\mathrm{pH}$ of paired beakers and $\mathrm{CV}$ of non-local beaker $\mathrm{pH}$ during Effector 2 sequence for trials associated with paired EMF exposure (Both On), single exposure (N-Local On Only), and non-exposure (Both Off).

be vulnerable to intervening or confounding variables that share the origin of the phenomenon. This could adversely affect the magnitude of the excess correlations. We would also expect that the manifestations of entanglement would be multiple and determined by local application geometry.

Previous experiments [8]-[11] have indicated that a specific sequence of circularly generated magnetic field with changing angular velocities are essential to produce the robust form of excess correlation we have measured in both pH-based and chemiluminescent reactions. They are the same patterns that have produced excess correlations between human brain activities as measured by quantitative electroencephalography [21]. The shared primary components have been brief exposure to circularly generated magnetic fields with decreasing angular velocity followed by increasing angular velocities. The occurrences of the excess correlations between the measurements of two loci (that have ranged from $10 \mathrm{~m}$ to $6000 \mathrm{~km}$ ) only become apparent in the second component of the exposure sequence. The duration of the maximum excess correlation is usually limited to about $8 \mathrm{~min}$. The specificity of the type of field and limit of the duration of "excess correlation" strongly counter-indicate the presence of equipment or procedural confounding variables.

In the present experiment we employed the same toroids and the same generating equipment that produced very conspicuous excess correlations in specific frequency bands within specific regions of paired human brains separated by $6000 \mathrm{~km}$. The parity in correlations in shifts in $\mathrm{pH}$ when measured at the two sites sharing the same field sequences when only one of the sites was injected with a proton donor was apparent. This also replicates our initial results [11] for this paradigm. The excess correlation did not occur when measurements were taken without the shared fields and was not evident when only one of the two sites were exposed to the magnetic field configurations.

Although the magnitudes of the excess correlations between two loci separated by $1 \mathrm{~m}$ (as was the case in previous experiments) and $100 \mathrm{~m}$ (as was the case in the present experiment) were comparable, these values for the $10,000 \mathrm{~m}$ distance were more variable. We suggest that the source of this dilution is not the limitation of the excess correlation but the involvement of superimposed variables that may comprise the bases for the type of excess correlation or a component of the bases. The candidate that emerged, although there could be many others, was the global geomagnetic activity at the time of the measurement. The excess correlation was attenuated when the global geomagnetic activity was K-4 or greater. When the global geomagnetic field was very quiet, 
that is there was less than $10 \mathrm{nT}$ variations of the most disturbed component during the time of the initiation and measurement of the excess correlation, the magnitudes of these correlations were largest.

The physical bases for this effect must still be discerned. However one of the effects of engaging the double-toroidal magnetic fields with this particular point duration of $3 \mathrm{~ms}$ is a discrete attenuation of between 1 and $5 \mathrm{nT}$ in the east-west component of the static geomagnetic field. Rouleau and Persinger [20] had measured this shift in this component of the geomagnetic field approximately 40 to $60 \mathrm{~s}$ before the onset of the first field associated with the excess correlation. This could indicate a temporally non-linear effect. They showed that this "antecedent" shift was within the range of the time constant obtained by dividing the rotational velocity of the earth at the latitude at which their measurements were by the value for $\mathrm{g}\left(9.8 \mathrm{~m} \cdot \mathrm{s}^{-2}\right)$. The potential role of Coriolis-like forces was also considered.

Rouleau and Persinger [20] calculated the energy of the inertial framework by multiplying the mass of the earth $\left(5.98 \times 10^{24} \mathrm{~kg}\right)$ and the square of the rotational velocity at the latitude of measurement $\left(4.63 \times 10^{2} \mathrm{~m} \cdot \mathrm{s}^{-1}\right)$. The value was $1.27 \times 10^{30} \mathrm{~J}$ and when divided by the surface area of the earth $\left(5.1 \times 10^{14} \mathrm{~m}^{2}\right)$ resulted in $0.25 \times$ $10^{16} \mathrm{~J} \cdot \mathrm{m}^{-2}$. When applied to the cross-sectional area occupied by an electron $\left(6.15 \times 10^{-30} \mathrm{~m}^{2}\right.$ per electron) the average energy for that space would be $1.5 \times 10^{-14} \mathrm{~J}$ per electron. This value is the same order of magnitude as the mass equivalence of an electron.

Hunter et al. [19] considered how the Earth's polarized electrons might be employed to study anomalous long-range spin-spin interactions. Another possible source for spin-spin interactions over long distances is the "unparticle" which can be characterized by an energy scale rather than mass. They estimated there are about $10^{49}$ unpaired electron spins in the earth; about $10^{42}$ become polarized antiparallel to the earth's magnetic field. Assuming the Earth's mass is $5.9 \times 10^{24} \mathrm{~kg}$, there would $2 \times 10^{17}$ polarized electrons per kg. Within our water samples of $50 \mathrm{cc}(2 \times 25 \mathrm{cc})$ there would have been $10^{16}$ polarized electrons. This is an interesting value because the numbers of electrons in $1 \mathrm{M}$ of water around $\mathrm{pH} 7\left(10^{-7} \mathrm{M}\right)$ when each Mole contains $6.023 \times 10^{23}$ molecules would converge with this value. Hence the number of these polarized electrons would be similar in magnitude to the hydronium ions that have been shown to be candidates for experimental entanglement in our other experiments [11].

Persinger and Rouleau [20] also found that the volume occupied by the magnetic energy from the averaged 1 - $5 \mathrm{nT}$ field shift in the east-west component would have displayed a linear distance of $\sim 21 \mathrm{~cm}$. This value not only corresponded with the approximate width of the toroid but exhibited the conspicuous concurrence with the neutral hydrogen line of $1.42 \mathrm{GHz}$. The involvement of the hydrogen line with excess correlation and entanglement has been suggested previously based upon other approaches [22].

Consideration of the circumference of the toroid as a mandatory component of the effect was indicated by calculating its inductance. The inductance of each toroid, which were the same ones employed in the present study, was $\sim 3 \times 10^{-5} \mathrm{H}$. The product of inductance $\left(\mathrm{kg} \cdot \mathrm{m}^{2} \cdot \mathrm{A}^{-2} \mathrm{~s}^{-2}\right.$ ) unit charge $(\mathrm{A} \cdot \mathrm{s})$ and the square of the frequency $\left(\mathrm{s}^{-2}\right)$ of the hydrogen line resulted in $9.4 \times 10^{-6} \mathrm{~V}$. The equivalent energy by multiplying by the unit charge would be the quantity of energy $1.5 \times 10^{-24} \mathrm{~J}$. When divided by Planck's constant $\left(6.626 \times 10^{-34} \mathrm{~J} \cdot \mathrm{s}\right)$ the frequency is within measurement error of $1.42 \times 10^{9} \mathrm{~Hz}$, the neutral hydrogen line.

The role of subtle gravitational factors also emerged. According to dimensional analyses the product of $G$ $\left(\mathrm{m}^{3} \cdot \mathrm{kg}^{-1} \cdot \mathrm{s}^{-2}\right)$, magnetic flux density $\left(\mathrm{kg} \cdot \mathrm{A}^{-1} \cdot \mathrm{s}^{-2}\right)$ and unit charge $(\mathrm{A} \cdot \mathrm{s})$ would be a cubed velocity term. Assuming the magnitude of the increment of change in the E-W geomagnetic field shift from our toroidal system, the value results in a velocity of $6.84 \cdot 10^{-13} \mathrm{~m} \cdot \mathrm{s}^{-1}$. The frequency obtained by dividing by the radius of the classic electron $\left(2.28 \times 10^{-15} \mathrm{~m}\right)$ would be roughly $3 \mathrm{msec}$, reflecting the point duration of the applied fields.

Provided that the intensity of the applied magnetic field capable of eliciting excess correlation operates in the nanoTesla range we may be able to demonstrate a convergence within the confines of the entire set (the Universe). The quotient of the inherent energy of the electrons $\left(1.5 \times 10^{-14} \mathrm{~J}\right.$ per electron) and the intensity of the applied field $\left(5 \times 10^{-9} \mathrm{~kg} \cdot \mathrm{A}^{-1} \cdot \mathrm{s}^{-2}\right)$ results in a magnetic moment of approximately $3.0 \times 10^{-6} \mathrm{Am}^{2}$. If we divide this resultant magnetic moment by the unit charge $\left(1.6 \times 10^{-19}\right.$ As) we end up with a magnetic diffusivity constant of $1.88 \times 10^{13} \mathrm{~m}^{2} \cdot \mathrm{s}^{-1}$.

Assuming the effect of excess correlation is directed through the interaction between energy and polarized electrons ( $\sim 10^{16}$ particles) the total charge, when multiplied by the unit charge, results in a total charge of $1.6 \times$ $10^{-3}$ As. If we divide the resultant charge by the calculated magnetic diffusivity, $1.88 \times 10^{13} \mathrm{~m}^{2} \cdot \mathrm{s}^{-1}$, we obtain a value of $8.51 \times 10^{-17} \mathrm{~A} \cdot \mathrm{m}^{-2}$. Again if we multiply this new value by the magnetic diffusivity we end up with a value of $1.6 \times 10^{-3} \mathrm{~A} \cdot \mathrm{s}^{-1}$. This value, and subsequent units reflect the necessary transformation to equate back- 
ground field intensities, whose magnitude are within the nanoTesla range, and the background radiant flux density of $10^{-12} \mathrm{~W} \cdot \mathrm{m}^{-2}$ and suggests a convergence between cosmological parameters [23].

We suggest that natural conditions upon the earth's surface may produce multiple, focal loci within which excess correlations would be "spontaneously" maximum. They would be analogous to conjugate points for geomagnetic latitudes within each hemisphere. They would be expected to be relatively narrow, perhaps a meter in width, similar to the unusual gradient areas reported in the 1940s by Solco Tromp [24]. The east-west components of the earth's magnetic field would be expected to be more or less equal if our experimental data can be generalized. Hence during specific geomagnetic activities the brains or people or the electrons within P-N junctions of electronic devices might be expected to show excess correlations.

However, if the two people operating the equipment or monitoring their cognitive experiences did not realize that other person was inducing changes within the equipment or perceiving specific events in the local environment the referent person might note unusual anomalies in the equipment or "anomalous" thoughts that could not be explained or accommodated by local factors. The source for the latter equipment anomalies or "non-self" experiences would originate within the other site but would exhibit superposition upon that site. We would not exclude symmetrical superposition for the two sites. The mechanism for the excess correlation would involve a type of entanglement employed in our experiments. That would require shared rotating magnetic fields with changing angular velocities for a period of several minutes within both loci. The probability of the experience would increase if the person sat quietly within minimum distraction from other energies.

Some of these coupled areas may involve sites which have been already designated by cultures that have lived in the area for centuries. We would expect them to be identified as unusual or "paranormal". During our years of measuring with MEDA vector fluxgate magnetometers we have encountered spaces in the natural environment where the geomagnetic field configurations that were effectively "vertical" patterns. Within these special places the very local inclination could shift almost 360 degrees thus producing a condition where rotational directions from superimposed much small perturbations might be guided. One application from our experimental model is that those places that share very similar specific geomagnetic intensities along the $\mathrm{X}, \mathrm{Y}$, and $\mathrm{Z}$ planes would display the capacity for excess correlation even with concomitant "noise" from geomagnetic perturbations.

Excess correlation between parity shifts in $\mathrm{pH}$ between two volumes of water separated by 1 to $10,000 \mathrm{~m}$ showed no evidence of attenuation with distance. The maximum strength of the excess correlations occurred after a primer field was activated. The correlates were displayed during the same interval after the "entanglement paradigm" was begun that has been expressed in other experiments. The discontinuity for the latter distance compared to 1 and $100 \mathrm{~m}$ was related to interference from the magnitude of global geomagnetic activity at the time of the experiment. The fact that disruption occurred could reveal more about the physical mechanisms of entanglement. The similarity of the intensity of the East-West geomagnetic vector when applied to the Earth's rotational parameters, inductance of the toroids, and electron drift indicates that the quantum of energy being "superpositioned" is equivalent to that of the electron. If natural equivalents of these rotational fields with changing angular velocities occur in any pair of separate spaces at any given time then excess correlation between similar processes should be possible.

Our findings suggest that local electromagnetic fields, global geomagnetic field conditions, and intrinsic $\mathrm{pH}$ variability contribute to the magnitude of excess correlation using toroidal coils which produce 1 - $5 \mathrm{nT}$ fluctuations within the E-W plane relative to magnetic North. These findings indicate that ferromagnetic shielding and other stabilizing technologies could be used in conjunction with excess correlation technologies to facilitate information or signal transfer.

\section{References}

[1] Arnesen, M.C., Bose, S. and Vedral, V. (2001) Natural Thermal and Magnetic Entanglement in the 1D Heisenberg Model. Physical Review Letters, 87, 017901.

[2] Aczel, A.D. (2002) Entanglement: The Greatest Mystery in Physics. Raincoast Books.

[3] Megidish, E., Halevy, A., Shacham, T., Dvir, T., Dovrat, L. and Eisenberg, H.S. (2013) Entanglement Swapping between Photons That Have Never Coexisted. Physical Review Letters, 110, 210403. http://dx.doi.org/10.1103/PhysRevLett.110.210403

[4] Fickler, R., Lapkiewicz, R., Plick, W.N., Krenn, M., Schaeff, C., Ramelow, S. and Zeilinger, A. (2012) Quantum Entanglement of High Angular Momenta. Science, 338, 640-643. http://dx.doi.org/10.1126/science.1227193 
[5] Olmschenk, S., Matsukevich, D.N., Maunz, P., Hayes, D., Duan, L.M. and Monroe, C. (2009) Quantum Teleportation between Distant Matter Qubits. Science, 323, 486-489. http://dx.doi.org/10.1126/science.1167209

[6] Basharov, A.M. (2002) Decoherence and Entanglement in Radiative Decay of a Diatomic System. Journal of Experimental and Theoretical Physics, 94, 1070-1079. http://dx.doi.org/10.1134/1.1493157

[7] Julsgaard, B., Kozhekin, A. and Polzik, E.S. (2001) Experimental Long-Lived Entanglement of Two Macroscopic Objects. Nature, 413, 400-403. http://dx.doi.org/10.1038/35096524

[8] Dotta, B.T. and Persinger, M.A. (2012) “Doubling” of Local Photon Emissions When Two Simultaneous, SpatiallySeparated, Chemiluminescent Reactions Share the Same Magnetic Field Configurations. Journal of Biophysical Chemistry, 3, 72. http://dx.doi.org/10.4236/jbpc.2012.31009

[9] Dotta, B.T., Murugan, N.J., Karbowski, L.M. and Persinger, M.A. (2013) Excessive Correlated Shifts in pH within Distal Solutions Sharing Phase-Uncoupled Angular Accelerating Magnetic Fields: Macro-Entanglement and Information Transfer. International Journal of Physical Sciences, 8, 1783-1787.

[10] Dotta, B.T., Karbowski, L.M., Murugan, N.J. and Persinger, M.A. (2013) Incremental Shifts in pH Spring Water Can Be Stored as "Space-Memory": Encoding and Retrieval through the Application of the Same Rotating Magnetic Field. NeuroQuantology, 11. http://dx.doi.org/10.14704/nq.2013.11.4.714

[11] Rouleau, N., Carniello, T.N. and Persinger, M.A. (2014) Non-Local pH Shifts and Shared Changing Angular Velocity Magnetic Fields: Discrete Energies and the Importance of Point Durations. Journal of Biophysical Chemistry. http://dx.doi.org/10.4236/jbpc.2014.52006

[12] St-Pierre, L.S., Parker, G.H., Bubenik, G.A. and Persinger, M.A. (2007) Enhanced Mortality of Rat Pups Following Inductions of Epileptic Seizures after Perinatal Exposures to 5 nT, 7 Hz Magnetic Fields. Life Sciences, 81, 1496-1500. http://dx.doi.org/10.1016/j.lfs.2007.09.013

[13] Dotta, B.T., Saroka, K.S. and Persinger, M.A. (2012) Increased Photon Emission from the Head While Imagining Light in the Dark Is Correlated with Changes in Electroencephalographic Power: Support for Bókkon's Biophoton Hypothesis. Neuroscience Letters, 513, 151-154. http://dx.doi.org/10.1016/j.neulet.2012.02.021

[14] Persinger, M.A., Dotta, B.T., Saroka, K.S. and Scott, M.A. (2013) Congruence of Energies for Cerebral Photon Emissions, Quantitative EEG Activities and 5 nT Changes in the Proximal Geomagnetic Field Support Spin-Based Hypothesis of Consciousness. Journal of Consciousness Exploration \& Research, 4.

[15] Vladimirskii, B.M. and Temur'Yants, N.A. (1996) Nuclear Magnetic Resonance in the Geomagnetic Field-The Possible Mechanism of Action of Weak Electromagnetic Fields on Biological and Physicochemical Systems. Biophysics, 4, 939-942.

[16] Persinger, M.A. and St-Pierre, L.S. (2014) Is There a Geomagnetic Component Involved with the Determination of G? International Journal of Geosciences, 5, 450-452. http://dx.doi.org/10.4236/ijg.2014.54042

[17] Korotaev, S.M., Morozov, A.N., Serdyuk, V.O., Gorohov, J.V. and Machinin, V.A. (2005) Experimental Study of Macroscopic Nonlocality of Large-Scale Natural Dissipative Processes. NeuroQuantology, 3, 275-294.

[18] Winch, D.E., Ivers, D.J., Turner, J.P.R. and Stening, R.J. (2005) Geomagnetism and Schmidt Quasi-Normalization. Geophysical Journal International, 160, 487-504. http://dx.doi.org/10.1111/j.1365-246X.2004.02472.x

[19] Hunter, L., Gordon, J., Peck, S., Ang, D. and Lin, J.F. (2013) Using the Earth as a Polarized Electron Source to Search for Long-Range Spin-Spin Interactions. Science, 339, 928-932. http://dx.doi.org/10.1126/science.1227460

[20] Rouleau, N. and Persinger, M. (2015) Local Electromagnetic Fields Exhibit Temporally Non-Linear, East-West Oriented 1 - 5 nT Diminishments within a Toroid: Empirical Measurement and Quantitative Solutions Indicating a Potential Mechanism for Excess Correlation. Journal of Electromagnetic Analysis and Applications, 7, 19-30. http://dx.doi.org/10.4236/jemaa.2015.72003

[21] Scott, M.A., Rouleau, N., Lehman, B.S., Tessaro, L.W.E., Juden-Kelly, L.M. and Persinger, M.A. (2015) Experimental Production of Excess Correlation Across the Atlantic Ocean of Right Hemispheric Theta-Gamma Power between Subject Pairs Sharing Circumcerebral Rotating Magnetic Fields (Part II). Journal of Consciousness Research \& Exploration, 6, 658-707.

[22] Persinger, M.A. and Lavellee, C.F. (2010) Theoretical and Experimental Evidence of Macroscopic Entanglement between Human Brain Activity and Photon Emissions: Implications for Quantum Consciousness and Future Applications. Journal of Consciousness Research \& Exploration, 1, 785-807.

[23] Persinger, M.A. (2014) Potential Gravitational-Solar Electromagnetic Spectral Radiance Interaction as the Source of the Earth’s Background Free Oscillations. International Letters of Chemistry, Physics, and Astronomy, 21, 11-14.

[24] Tromp, S.W. (1949) Psychical Physics: A Scientific Analysis of Dowsing, Radiethesia and Kindred Divining Phenomena. Elsevier, New York. 


\section{Submit or recommend next manuscript to SCIRP and we will provide best service for you:}

Accepting pre-submission inquiries through Email, Facebook, LinkedIn, Twitter, etc.

A wide selection of journals (inclusive of 9 subjects, more than 200 journals)

Providing 24-hour high-quality service

User-friendly online submission system

Fair and swift peer-review system

Efficient typesetting and proofreading procedure

Display of the result of downloads and visits, as well as the number of cited articles

Maximum dissemination of your research work

Submit your manuscript at: http://papersubmission.scirp.org/ 